\title{
CLASSIFICATION IN SCIENTIFIC AND TECHNICAL WRITING
}

\author{
Jelisaveta Šafranj (D), Marina Katić (D), Jelena Zivlak \\ University of Novi Sad, Faculty of Technical Sciences, Novi Sad, Serbia
}

\begin{abstract}
Classification is the rhetorical device closely related to formal definition. The first step in definition is to classify the term being defined. The term is placed in a group whose members have at least one outstanding characteristic in common. This member is differentiated from all other members of the class. In classification, however, the groups rather than individual members are differentiated. The reader is given the important information about the name of the class, the members of the class important for the discussion and basis for classification, often called criterion of difference. The basis of classification always reflects the particular purpose of the writer making the classification, and the basis relevant to one person may well be irrelevant to another.
\end{abstract}

Key words: classification, technical writing, English for Specific Purposes

\section{INTRODUCTION}

Classification is one of the most fundamental ways of organizing and explaining technical materials. The writer's subject is always different and comprises technical objects or incidents, ideas or sensory experiences, material properties or products of his imagination (Kieft, 2016). Thus, the only common thing is that all are capable of being classified. In classifying not only differences, but similarities are stated as well. In addition, in a class containing a large number of members, the writer chooses those members that are important to the subject under discussion and uses one or more characteristics he considers important as basis for classification (Herbert, 2018).

The purpose of classification is to increase the reader's understanding of a subject, but such an understanding is often only a preparation for writing a piece of exposition. It may serve as an outline, and often can provide the structure of discussion (Day and Gastel, 2016).

Classification can be divided into two groups: explicit and implicit depending on whether the information is stated in the form of classification, i.e., depending on whether the information is stated directly (explicit classification) or indirectly (implicit classification). In addition, classification can be complete or full and partial or incomplete depending on whether the criterion of difference is given or not. Also, classification can be simple or complex depending on the number of levels of generality given (Jordan, 2015).

\section{EXPLICIT CLASSIFICATION}

Explicit classification is easy for the reader to detect; the author tells the reader that he is going to classify. Such words as classify, group, categorize, divide, leave no doubt in the readers mind as to what is going on. The following examples illustrate explicit classification in EST.

Example 1. Explicit classification (simple)

Printing inks

Printing inks are principally made up of colorants (pigments, dyes), vehicles (binders), additives, carrier substances (solvents). Depending on the type of printing process, inks have largely variable flow properties which range from extremely thin (watery), through highly viscous, up to dry (powder-like). The ink transfer mechanism and the type of drying/fixing of the ink on the substrate principally determine the structure and the components of a printing ink (Kipphan, 2001).

In the above quoted example the writer provides the following important information: (1) the name of the class: printing inks, (2) the members, of the class: thin, highly viscous and dry inks; (3) the basis for classification: according to their flow properties. This classification is given on one level of generality and that is why it is considered simple; it is complete or full because it gives all necessary information (Murray and Moore, 2016). 
Explicit classification (complex)

Liquids

Liquids can be broadly classified as viscous liquids and thin liquids, depending on whether they offer a large or small resistance to motion relative to solid surface. Typical viscous liquids are thick oils and glycerine, while typical thin liquids are water and gasoline. The shear coefficient of viscosity of the typical viscous liquids are high, about 100 times higher than the shear coefficient of viscosity of the typical thin liquids (which, in turn, are about 10 times as high as those of gases). The thin liquids and gases are considered as fluids having very small (shear coefficient) viscosity (Owczarek, 2008).

The classification in the above example is given on two levels of generality, as can be seen from the following diagram:

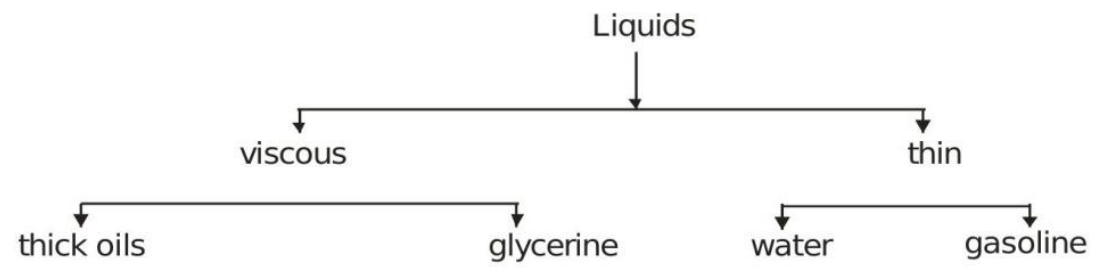

Figure 1: Complex explicit classification

For the first level of this complex explicit classification the writer differentiates the members of the main class (liquids) according to the resistance they offer to motion relative to a solid surface. The basis for classification of the members is not given.

As can be seen from the previous examples that the reader receives all three kinds of information which every complete classification should give:

a) the class,

b) the important members of the class,

c) the criterion of difference.

If the reader is given this information, the classification is considered complete. When the reader is given the class and the members, but not the criterion of difference, the classification is partial (Jordan, 2015). Sometimes the criterion of difference is left out on the assumption that it is obvious to the reader. Then the classification is partial.

Example 2. Partial classification in EST

Forms of Matter

All forms of matter can be grouped into two classes: solids and fluids. Webster's dictionary defines a fluid as a substance which yields to any force, however small, tending to alter its shape. In somewhat more technical terms a fluid may be described as a substance which, when at rest, cannot sustain a shear force, that is, a force exerted tangentially to the surface on which it acts. Solids, on the other hand, can withstand such forces, and although they may remain at rest, displacements or strains of the material result. A shear force can be sustained in a fluid, however, when relative motions between the particles of the fluid take place. Under ordinary conditions this distinction, between fluids and solids is readily apparent for such materials as water, air, or steel. However, it is difficult to determine whether some substances should be classified as solids or fluids (Owczarek, 2008).

But not always does the writer give classifying information in a well organized way as can be seen from the stated examples. The following example show a conceptual paragraph in which an explicit (partial) classifying information is given in a reversed order: 
Example 3. Explicit out of order classifying information (complex)

Drying Methods

There are two chemical drying methods:

a) oxidation is a polymerization of the binder - vehicle of printing ink,

b) polymerization by cross-linking through UV radiation or cross-linking through electron beams

The above mentioned two types of drying methods are chemical methods. Physical method or absorption is Penetration achieved by the interaction of printing ink and substrate.

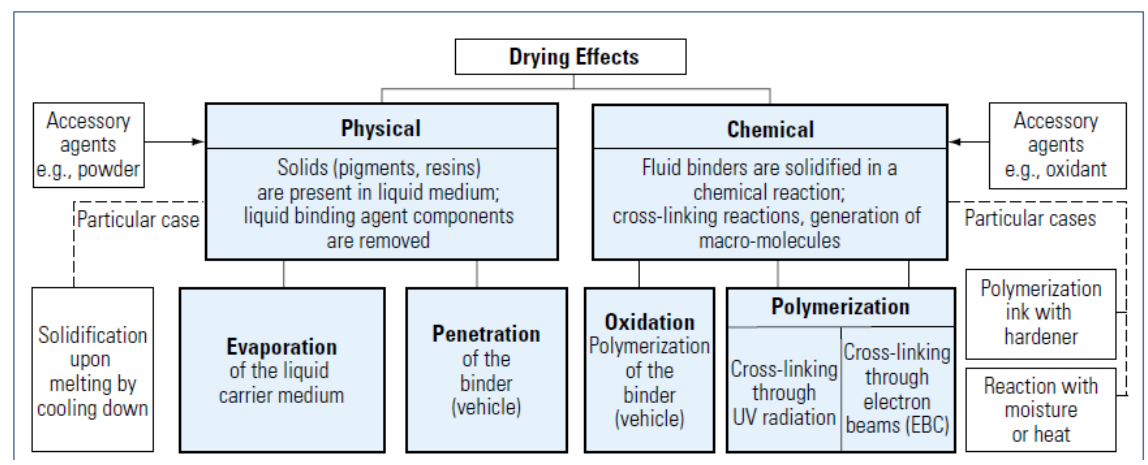

Figure: 1.7-1 Drying processes (Kipphan, 2001)

From the above drawn diagram the two levels of generality of this complex explicit classification can be noticed very easily. But for the reader to be able to abstract and reorder the information given, in the form of the above drawn diagram is not easy, unless he possesses at least elementary technical knowledge of the particular subject (Kipphan, 2001).

\section{IMPLICIT CLASSIFICATION}

If the reader can take information not stated as classification and arrange it into the form of classification, then there is obviously classificatory information in the writing. This type of classification is called implicit classification. In a paragraph containing implicit classification, a statement containing classification information is itself seldom the generalization or even a part of the generalization. Rather the implicit classifying information is buried in the information which supports and develops the core generalization. Thus, implicit classifying information should be extracted and reordered from the supporting information. The primary purpose of a paragraph containing implicit classifying information is not usually Classification but rather Definition, Description or Presenting information on experimental procedure. Like explicit classification, implicit classification is found in both simple and complex form.

Example 4. Implicit classification (simple)

A distinction is made between technologies requiring a master, conventional printing, and so called nonimpact printing (NIP) technologies which do not require a printing plate. Printing technologies requiring a printing plate are technologies like lithography (offset), gravure, letterpress, and screen printing. The most common NIP technologies are electrophotography and ink jet (Kipphan, 2001).

In the above quoted paragraph the writer implicitly classifies printing technologies. After a careful reading the reader can draw the following diagram: 


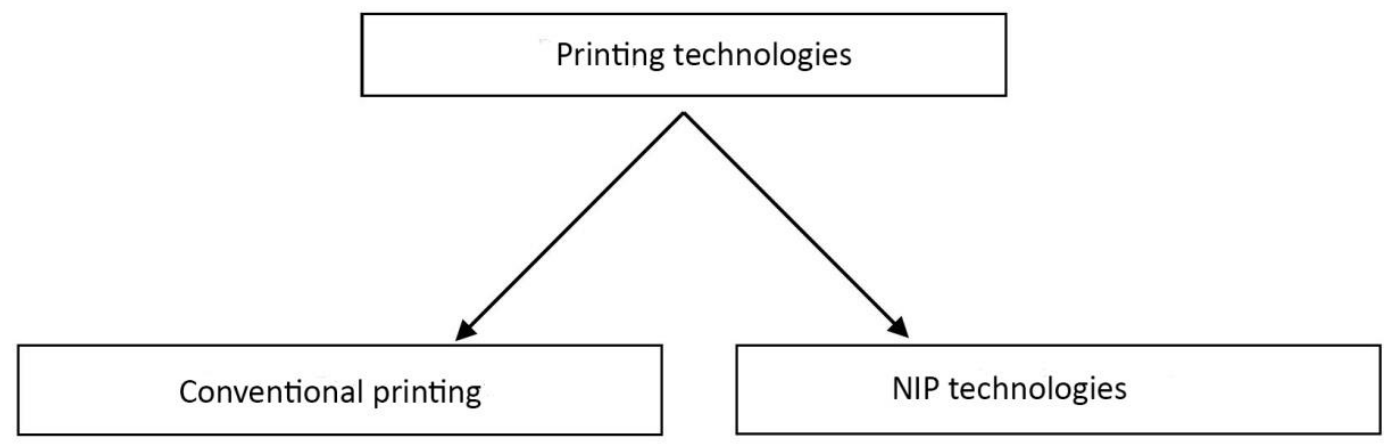

Figure 2: Simple implicit classification

The above implicit classification is a one-level classification and it is considered simple.

Example 5. Implicit classification (complex)

Printing inks

Printing inks are principally made up of colorants (pigments, dyes), vehicles (binders), additives, carrier substances (solvents). Depending on the type of printing process, inks have largely variable flow properties which range from extremely thin (watery), through highly viscous, up to dry (powder-like). The ink transfer mechanism and the type of drying/fixing of the ink on the substrate principally determine the structure and the components of a printing ink. Colorants are divided into: pigments (organically or inorganically coloured, white, or black substances that are insoluble in the ink vehicle.) These are solid particles and/or molecular agglomerates that must be held in suspension in the base liquid), dyes (organic compounds that are dissolved in the system during application) which are present in molecular form.

Pigments consist of molecules that are cross-linked with one another as crystals. Normally pigments have a particle size of 0.1-2 $\mu \mathrm{m}$. A pigment particle can consist of several million molecules. Only around $10 \%$ of the molecules lie on the surface, and it is only these molecules and a few underneath that can absorb light. Pigments disperse light and, as such, are opaque. They have a wide absorption band and are therefore not as "pure" as dyes, which possess an extremely narrow absorption band.

Dye molecules are surrounded by solvents (base liquid), so that almost every molecule can absorb photons, which leads to higher colour intensity and more luminous colours. Dyes have a larger range of colours. They are naturally transparent since the molecules are significantly smaller than the visible wave length of 380 $\mathrm{nm}$.

Pigments always require a vehicle for binding them to the substrate while dyes in most applications connect themselves directly to the substrate surface. The disadvantage with most dyes is their limited light-fastness (oxidation leads to bleaching). With respect to light fastness and stable ink impression, pigment-based inks are advantageous. Pigments as base materials are basically cheaper than dyes yet require greater expenditure when being processed into ink: dispersing agents must be added to pigments so that they do not agglomerate. Dyes are, in contrast, dissolved and do not deposit themselves in the liquid. Printing inks normally contain pigments. The most important exception is currently still those inks used in ink jet technology, but even here the trend is towards pigments (better light-fastness, less bleeding on the paper). The pigment content, depending on the colour tone, is between $5 \%$ and approximately $30 \%$. Those organic pigments which give the printing inks (process inks) the desired colours (hue) are most important for the printing industry. They can be grouped into the two main categories of chromatic pigments and black pigments.

The main inorganic pigments are: white pigment (e.g., titanium dioxide), metal effect pigment (gold and silver bronzes), pearlescent pigment, fluorescent pigment (for daylight luminous colours).

Binders. In conventional printing processes, binders are normally resins dissolved in mineral oil. The pigments are finely dispersed in the binder. The pigment particles are then enclosed by a binder shell. The shell protects the finely dispersed particles from associating into agglomerates and being deposited. The binders dry (harden) on the substrate and thereby bind the pigments.

Additives. The type of additive is dependent on the respective printing process. Additives are added to the ink in particular to influence drying, flow behaviour, and abrasion resistance. 
Carrier substances. Carrier substances for the colorants in conventional printing processes are on the one hand thinning agents such as mineral oils and on the other, insofar as available, solvents (e.g., toluene in gravure printing) (Kipphan, 2001).

The core generalization of the above paragraph is stated in the first sentence. Buried in the supporting details is information that can be put in the form of an explicit classification. From the two sentences of this paragraph, two-level classification can be extracted and reordered in the form of the following diagram (Kipphan, 2001):

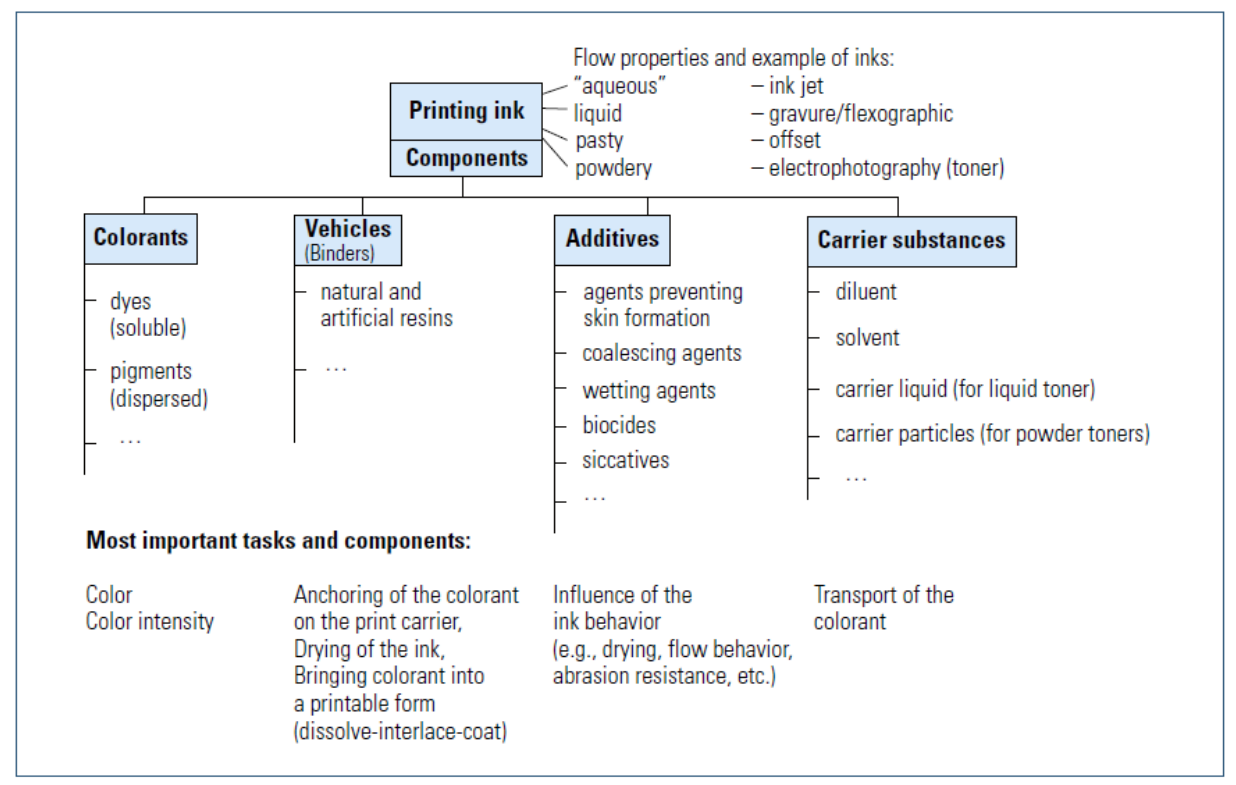

Figure 3: Complex implicit classification on two levels (Kipphan, 2001)

\section{CONCLUSION}

The purpose of the basis for classification is to tell the reader in what essential way each member of the class differs from each other member of the class. Therefore, it is a major meaning component in a statement of classification. It should be noted that statements of classification also contain by implication, as a rule, basis for similarity. Obviously, the given members of a class have one or more areas of similarity or they would not be members of the same class. A named class is not a mere sum of the members that fall within the class. It is, rather, the idea, the concept, of the qualities that any particular member should have in order to fall within the class. In other words, a class is determined by a complex of significant characteristics shared by all members of the class.

Since the rhetorical device of classification is man-made a thing may be, and usually is, classified in a number of ways depending on the basis on which the classification is made. The basis of classification will always reflect the particular purpose of the writer making classification, and basis relevant to one person may well be irrelevant to another.

\section{REFERENCES}

[1] Day, R. A., Gastel, B.: "How to write and publish a scientific paper", 6th ed., (Cambridge University Press Cambridge, 2016.).

[2] Herbert, A. J.: "The Structure of Technical English", (Longmans Green \& Co. Ltd, London, 2018).

[3] Jordan, R. R.: "English for Academic Purposes: A Guide and Resource Book for Teachers", (Cambridge University Press Cambridge, 2015.).

[4] Kieft, M.: "The Effects of Adapting Writing Instruction to Students' Writing Strategies", (University of Amsterdam, Amsterdam, 2016.).

[5] Kipphan, H.: "Handbook of Print Media", (Springer, Berlin, Heidelberg, New York, 2001.), page 130, $131,211,166,681$. 
[6] Murray, R., Moore, S.: "The Handbook of Academic Writing: A fresh approach", (Open University Press, Maidenhead, 2016.).

[7] Owczarek, J.A.: "Introduction to Fluid Mechanics", (International Textbook Com., Scranton, Pennsylvania, 2008.), page 17, 43.

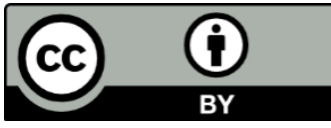

(C) 2020 Authors. Published by the University of Novi Sad, Faculty of Technical Sciences, Department of Graphic Engineering and Design. This article is an open access article distributed under the terms and conditions of the Creative Commons Attribution license 3.0 Serbia (http://creativecommons.org/licenses/by/3.0/rs/). 